\title{
Bonos verdes versus bonos convencionales: ¿existe una demanda diferenciada en México?
}

\author{
Rogelio Arellano Cadena \\ Banco de México ${ }^{1}$ \\ Lucía Pérez Delgado \\ Universidad Anáhuac México
}

Artículo recibido el 11 de enero y aprobado el 23 de mayo de 2019

\section{Resumen}

¿Es la demanda de bonos verdes diferente a la demanda de bonos convencionales? A partir del estudio seminal de Fama y French (2007) quienes consideraron que la demanda de activos financieros, además de criterios de rendimiento y riesgo, podría explicarse por motivos no pecuniarios, diversos trabajos han intentado responder a esta pregunta (Asian Bond Monitor, 2018; Ehlers y Packer, 2017; Reboredo, Quintela y Otero, 2017; Östlund, 2015, y Zerbib, 2016, 2019). La mayoría de los resultados indican que los bonos verdes otorgan un rendimiento inferior a aquel pagado por los bonos convencionales. Ello hace concluir que si existe una demanda diferenciada de este tipo de bonos. Hasta ahora, no se cuenta con estudios que realicen esa comparación para el caso de México.

El presente análisis pretende cerrar esa brecha, intentando identificar si existe una diferenciación por parte de los inversionistas en México entre su demanda de un bono verde y de un bono convencional. Para ello, se utilizan las posturas de compra (ask price) para dichos bonos emitidos por la misma institución, ya sea en la misma fecha o con el mismo plazo de vencimiento. Los resultados sugieren que la distribución de las posturas de compra difiere entre uno y

1 Las opiniones aquí expresadas son responsabilidad del autor y no reflejan la postura del Banco de México. 
otro tipo de bono y que, cuando los bonos tienen el mismo plazo de vencimiento, los inversionistas están dispuestos a aceptar rendimientos ligeramente inferiores en bonos verdes.

Palabras clave: bonos verdes, bonos convencionales, riesgos ambientales. Clasificación JEL: Q51, Q54. 


\begin{abstract}
Is the demand for green bonds different from the demand for conventional bonds? Previous analysis that compare financial returns between green and conventional bonds (Asian Bond Monitor, 2018; Ehlers and Packer, 2017; Reboredo, Quintela y Otero, 2017; Östlund, 2015, and Zerbib, 2016, 2019) show, in most cases, that green bonds have a lower return than conventional bonds. No study comparing such bonds has yet been done for Mexico.

This study aims to close such gap, intending to identify if investors have a differentiated demand for green bonds with respect to comparable conventional bonds. Contrary to previous analysis that compare observed returns, we use the ask prices for green and conventional bonds issued either on the same date or with the same maturity by the same institution. The results suggest that the distribution on ask prices differ between green and conventional bonds. Additionally, there is a negative difference in their mean returns for bonds with the same maturity.
\end{abstract}

Keywords: green bonds, conventional bonds, environmental risks. JEL Classification: Q51, Q54. 


\section{Introducción}

El estudio del impacto que el calentamiento global y, en general, que las modificaciones en el medio ambiente ejercen en la economía es relativamente reciente: los análisis más detallados se han realizado en la última década. ${ }^{2}$ Estos estudios se han enfocado, en particular, en señalar las probables consecuencias de mantener temperaturas promedio superiores a $2{ }^{\circ} \mathrm{C}$ en relación con la época preindustrial. Es de notar que el análisis sobre la participación que el sector financiero puede tener para mitigar dichos impactos o los riesgos que el medio ambiente puede inducir en la estabilidad financiera es aún más reciente. En el primer caso, destaca el papel que los instrumentos (bonos, créditos e instrumentos mixtos) denominados «verdes» pueden tener para fondear proyectos amigables con el medio ambiente y que, a la vez, sean atractivos para los inversionistas. ${ }^{3}$

En el ámbito multilateral, los trabajos del Grupo Intergubernamental de Expertos sobre el Cambio Climático han sido fundamentales para destacar las consecuencias adversas del cambio climático y sugerir acciones para enfrentarlo. Así por ejemplo, en su análisis sobre sus efectos efectuado a principios de la presente década, el Grupo de Expertos (2013) destacó, entre otros puntos, que:

- El calentamiento en el sistema climático es inequívoco y, desde la década de 1950, muchos de los cambios observados no han tenido precedentes en los últimos decenios.

- La producción de cereales se reduce un $5 \%$ aproximadamente por cada grado de aumento en la temperatura.

- Entre 1901 y 2010, el nivel medio del mar en el mundo aumentó $19 \mathrm{~cm}$. Se prevé una elevación media del nivel del mar de entre 24 y $30 \mathrm{~cm}$ para 2065 y entre 40 y $63 \mathrm{~cm}$ para 2100 .

- Las emisiones mundiales de dióxido de carbono $\left(\mathrm{CO}_{2}\right)$ han aumentado casi $50 \%$ desde 1990 . Entre 2000 y 2010 se produjo un incremento de las emisiones mayor que en las tres décadas anteriores.

2 Son de destacar los estudios del Banco Mundial (2018), y del Banco Mundial e International Financial Corporation (2016), el Foro Económico Mundial (2018), el Grupo Intergubernamental de Expertos sobre el Cambio Climático (IPCC, por sus siglas en inglés, 2013), el Instituto Nacional de Ecología y Cambio Climático (2014) y de la OCDE (2018).

3 En el caso de instrumentos financieros verdes, véase Comisión Europea (2016) y Banco Mundial e International Finantial Corporation (2016). Para el tema de riesgos ambientales y estabilidad financiera, consúltese Nieto (2017). 
- Las pérdidas anuales promedio causadas solo por tsunamis, ciclones tropicales e inundaciones alcanzan los cientos de miles de millones de dólares y exigen inversiones de unos 6000 millones de dólares anuales solo en gestión del riesgo de desastres. ${ }^{4}$

Estos hechos, por demás contundentes, impulsaron a que, en 2015, la Organización de las Naciones Unidas adoptara, entre los Objetivos Globales para el Desarrollo Sostenible, medidas urgentes para combatir al cambio climático y sus efectos. La meta es limitar el aumento de la temperatura media global a un máximo de $2{ }^{\circ} \mathrm{C}$ respecto a los niveles preindustriales. Como instrumento para alcanzar los Objetivos Globales, se realizó un compromiso de carácter financiero: movilizar, para el año 2020, 100000 millones de dólares anuales para ayudar a mitigar los desastres relacionados con el cambio climático. Por su parte, la Organización para la Cooperación y el Desarrollo Económicos (OCDE, 2017) estima que para 2030, y con el objetivo de cumplir con las metas establecidas, se requieren casi siete billones (millones de millones) de dólares para fondear proyectos de infraestructura amigable con el medio ambiente. Para el caso de México, la Corporación Financiera Internacional (2016) estimó un potencial de inversión en energía renovable del orden de 75000 millones de dólares.

De acuerdo a Zerbib (2016), el sector privado tiene los recursos financieros requeridos para facilitar la transición hacia una economía más amigable con el medio ambiente, complementando los fondos públicos disponibles. Un vehículo para lograr dicho objetivo lo constituyen, precisamente, los bonos verdes.

El concepto de «bono verde» tiene su origen en 2007, cuando el Banco de Inversión Europeo colocó el Climate Awareness Bond. A partir de entonces, la emisión de bonos verdes ha tenido un desarrollo dinámico. Ha sido utilizado por organismos multilaterales (empezando por el Banco Mundial en 2008), gobiernos subfederales (el primero en Gotemburgo en 2013), corporativos no financieros (el primer bono verde de este tipo fue emitido por la empresa Vasakronan, en Suecia en 2013), bancos de desarrollo nacionales (siendo el primero en su tipo el banco alemán KfW, en 2014), y naciones (Polonia fue el primer país en emitir un bono soberano verde en 2016). ${ }^{5}$

4 Más recientemente, el Banco Mundial (2018) — entre otras instituciones - ha analizado el posible impacto del cambio climático sobre los flujos migratorios: sin la implementación de acciones para mitigar el cambio climático, el Banco Mundial estima que alrededor de 143 millones de personas de las regiones de Latinoamérica, África subsahariana y el Sur de Asia (aproximadamente el $2.8 \%$ de la población de dichas regiones) estarían forzadas a migrar debido a la disminución de la disponibilidad de agua y a la reducción de la productividad en sus cosechas.

5 Para un desarrollo detallado de la evolución de la utilización de bonos verdes a nivel internacional, tema que va más allá del objetivo de este documento, 
Precisamente, la fuerte penetración de bonos denominados como verdes en el mercado financiero internacional incidió en que, para fortalecer la confianza de los inversionistas en este instrumento y evitar que los recursos obtenidos por esta vía fueran asignados a proyectos «no verdes», la Asociación Internacional de Mercados de Capital (ICMA por sus siglas en inglés) desarrollara los «Principios de Bonos Verdes». En estos lineamientos se establecieron principios rectores en cuatro aspectos: a) uso de los recursos provenientes de la emisión; b) proceso de evaluación y selección de los proyectos a fondear; c) administración de los recursos y d) reportes periódicos. Estos Principios definieron bono verde como «cualquier tipo de bono en el que los fondos se aplicarán exclusivamente para financiar o re-financiar, en parte o en su totalidad, Proyectos Verdes elegibles, ya sean nuevos y/o existentes» (Asociación Internacional de Mercados de Capital, 2014).

En México, los principios de la ICMA fueron adoptados por el Consejo Consultivo de Finanzas Climáticas, ${ }^{6}$ que en 2016 emitió los «Principios de Bonos Verdes MX». Con ellos se establecieron lineamientos para el proceso de emisión de este tipo de bonos con el objetivo de dar confianza a los potenciales compradores sobre el beneficio ambiental asociado a los proyectos financiados.

\section{Marco conceptual: bonos verdes}

Los bonos verdes tienen características muy similares a las de los bonos convencionales, excepto porque los fondos recaudados se dirigen exclusivamente a la inversión de proyectos que favorecen al medio ambiente o que detienen su deterioro. Existen, no obstante, algunas diferencias, como la menor liquidez de los bonos verdes y los mayores costos asociados a la comprobación de que, en efecto, los fondos recaudados se dirijan hacia proyectos verdes.

véase Federal Ministry for Economic Cooperation and Development (2018), Moid (2018), Organización para la Cooperación y el Desarrollo Económico/ Asociación Internacional de Mercados de Capital (2016) y Sustainable Banking Network, World Bank International Finance Corporation (2018).

6 Este Consejo se integra por la Bolsa Mexicana de Valores, la Asociación de Bancos de México, la Iniciativa de Bonos Climáticos (Climate Bond Initiative), la Asociación Mexicana de Instituciones Bursátiles, la Asociación Mexicana de Administradores de Fondos para el Retiro, la Asociación Mexicana de Instituciones de Seguros, la Asociación Mexicana de Asesores Independientes de Inversiones, bancos de desarrollo, bancos multilaterales, inversionistas institucionales, emisoras y calificadoras. 
Sin embargo, cuando un bono verde y un bono convencional son colocados por el mismo emisor, sus perfiles de riesgo son prácticamente idénticos, toda vez que, inclusive, el pago de intereses y capital de los bonos verdes puede provenir de recursos generados por el emisor.

Para los emisores de un bono verde, los posibles beneficios son (Mexi$\mathrm{CO}_{2}, 2017$, Moid, 2017):

- Imagen y visibilidad ante sus inversionistas, manifestando un compromiso creíble sobre una estrategia ambiental;

- Atracción de nuevos inversionistas con estrategias sustentables que buscan activos con impactos ambientales positivos; 7

- Aumento en la confianza e interés del inversionista al ofrecer transparencia y certeza sobre el uso de los recursos;

- Facilitación del financiamiento del proyecto al adecuar su duración con la del bono.

Por su parte, los posibles beneficios para los inversionistas son:

- La alineación de sus intereses como inversionistas «ambientalmente responsables» con la compra de activos verdes que, inclusive, puede ser un mandato de su Consejo;

- Diversificación de portafolios.

En materia de rendimientos, los proyectos verdes pueden, por una parte, estar asociados a un menor rendimiento, toda vez que tienden a enfrentar mayores costos de producción y de desarrollo tecnológico. Por la otra, dado que los activos asociados a energías renovables e inversiones amigables con el medio ambiente han ganado terreno - tanto competitiva como tecnológicamente - en relación con proyectos relativos a combustibles fósiles o con impactos adversos al medio ambiente, los rendimientos de los instrumentos asociados a proyectos verdes pueden ser mayores que los vinculados con otro tipo de proyectos.

7 Cabe mencionar que se han desarrollado varias iniciativas para promover la asignación de activos financieros hacia inversiones verdes. Una de ellas, es el Montreal Carbon Pledge, iniciativa que empezó en 2014 y en la que más de 120 inversionistas internacionales con activos administrados con un valor superior a los 10 billones de dólares se comprometieron al desarrollo del mercado de bonos verdes y a medir e informar sobre la huella de carbón de sus inversiones. 
En México, la primera emisión de bonos verdes se efectuó a finales de 2015, con la colocación de un bono por 500 millones de dólares de Nacional Financiera para financiar proyectos de energía eólica. Hasta octubre de 2018, se habían realizado en México trece colocaciones de bonos verdes/sustentables/sociales, por un monto aproximado de \$166000 millones (tabla 1). Las mayores colocaciones correspondieron al Grupo Aeroportuario de la Ciudad de México, que en 2016 emitió bonos verdes por un valor de 2000 millones de dólares y, un año después, por 4000 millones de dólares.

Como puede apreciarse en la tabla 1, las colocaciones de bonos verdes han sido muy variadas, tanto en monto (desde 600 hasta 6000 millones de pesos) como en plazo (desde tres hasta 25 años). No obstante, destaca que en la mayoría de las emisiones se ha registrado una sobredemanda, medida por la relación entre el monto solicitado por los inversionistas y el convocado por la institución o empresa colocadora. Es de subrayar, en particular, la sobredemanda en las emisiones de bancos de desarrollo (NAFIN, cinco y tres veces el monto convocado, Banobras, 5.2 veces) y por el Grupo Aeroportuario de la Ciudad de México (GACM, 6.5 veces), cifras que sugieren el interés del público inversionista por este tipo de bonos. Ahora bien, la sobredemanda referida no es necesariamente un indicador de que dicho bono, por el hecho de catalogarse como «verde», sea preferido a un bono convencional. Para ello sería necesario comparar, al menos, la sobredemanda de bonos convencionales colocados por oferentes con la misma calificación de riesgo, con el mismo plazo y en el mismo día. Tomando esto en consideración, con el propósito de identificar si existe o no una diferenciación de los inversionistas entre un bono verde y un bono que no tiene esa categoría (bono convencional), en las siguientes secciones del documento se hace un análisis al respecto. Primero, se resumen los principales estudios sobre el tema, los cuales se enfocan en comparar el diferencial de rendimiento de un bono verde con uno convencional. Posteriormente se presenta un análisis de caso para México. 


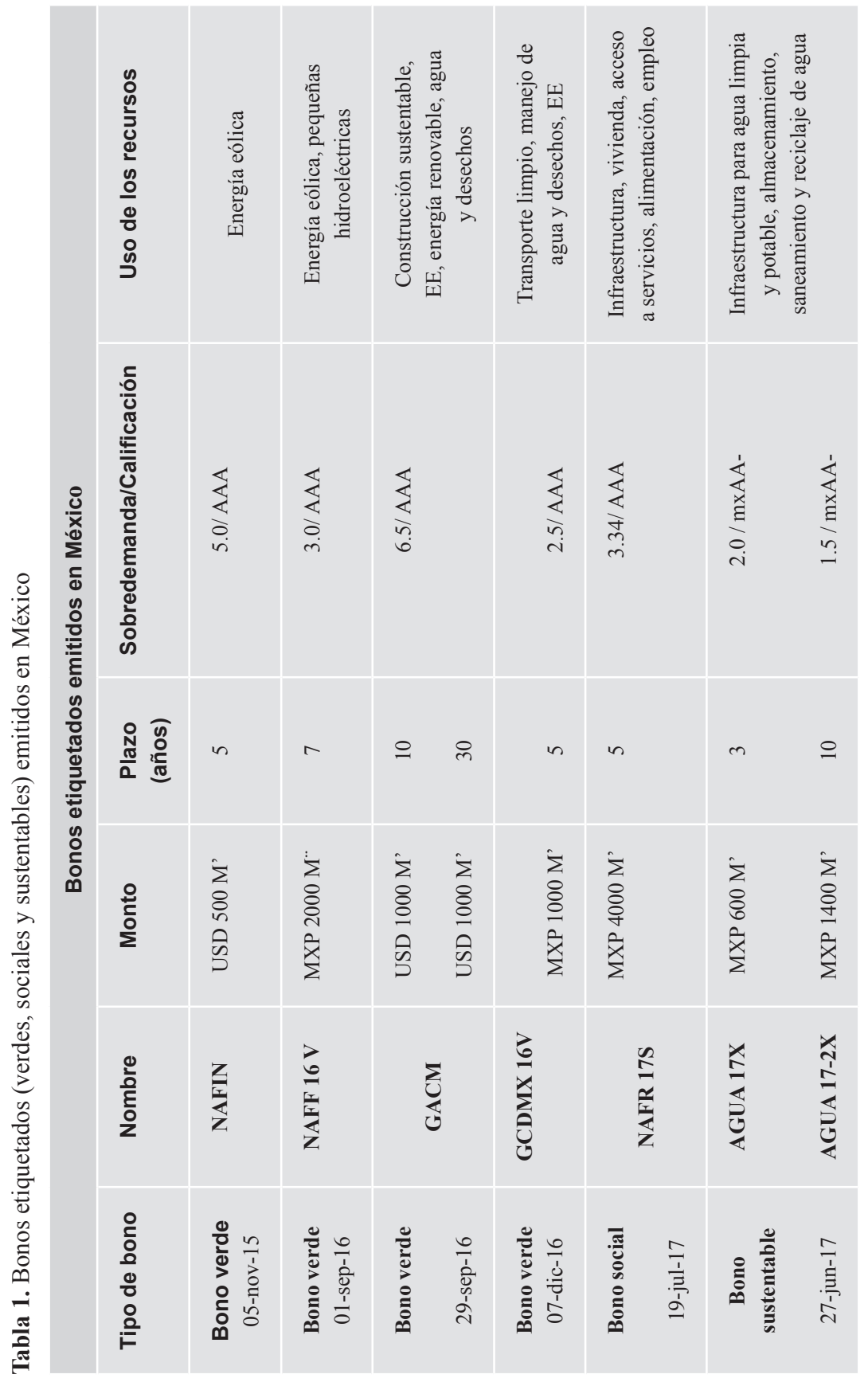



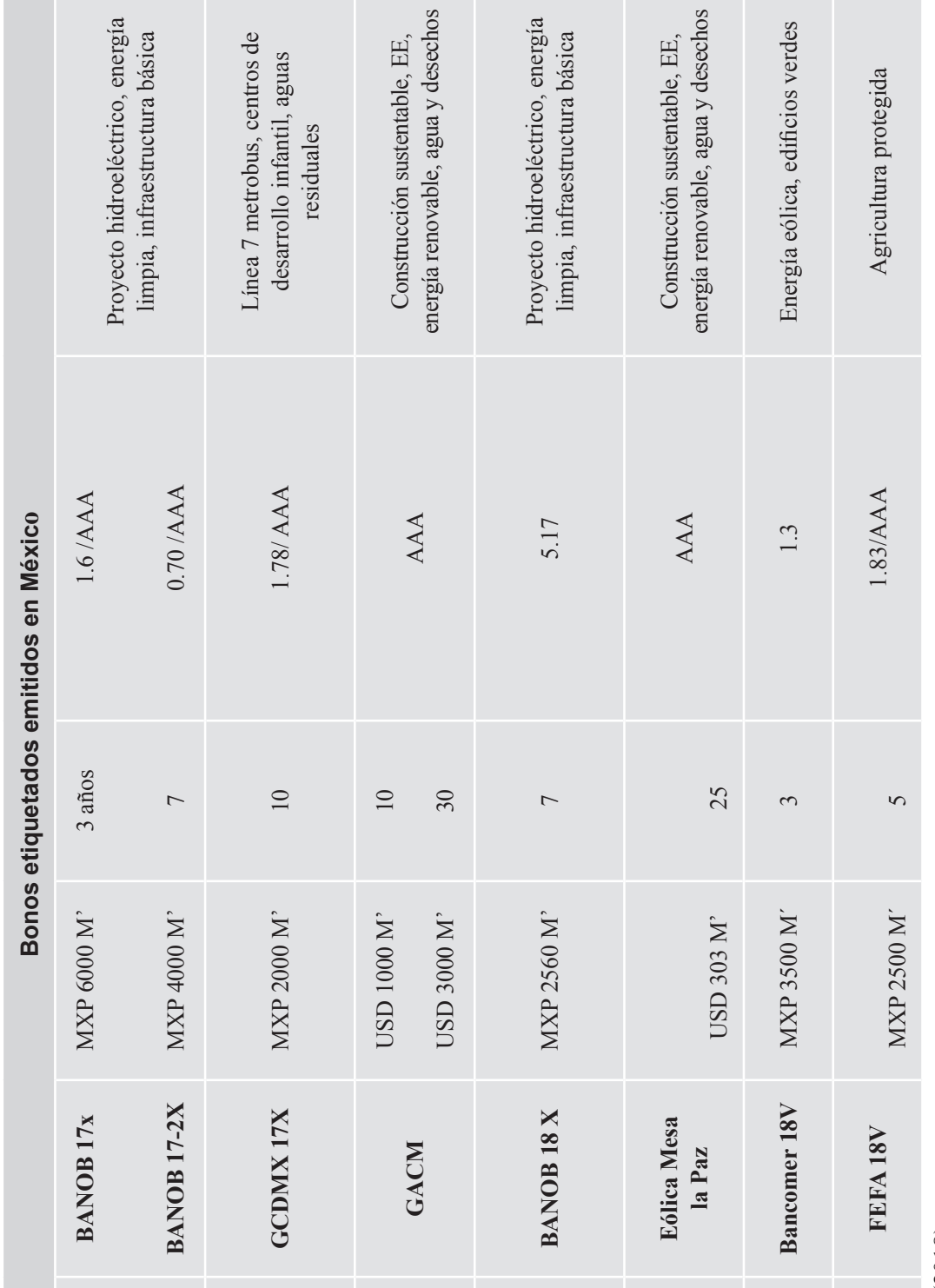

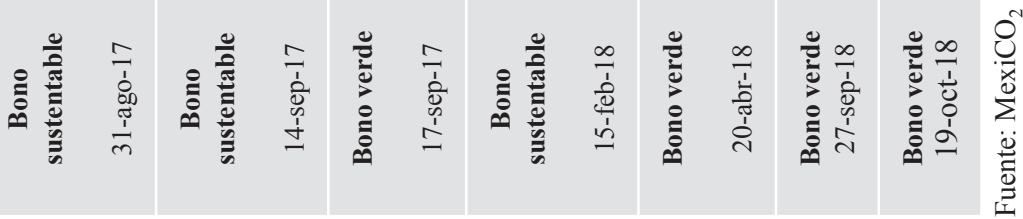




\section{Marco teórico}

En los modelos de valuación de precios de activos, uno de los supuestos utilizados es que los inversionistas consideran primordialmente información de rendimientos y riesgos para la elección de sus portafolios. No obstante, Fama y French (2007) relajaron estos supuestos al estimar que los activos financieros pueden ser demandados por otras razones, considerándose no solo «bienes de inversión» sino también «bienes de consumo». Ello ocurre, por ejemplo, cuando un inversionista, por su «deseo de pertenecer» a la compañía, tiene activos de su empleador más allá de razones de rendimiento y riesgo, o cuando prefiere invertir en activos de su país o de su localidad, aunque el rendimiento sea inferior a otro tipo de activos. Un ejemplo adicional ocurre cuando el inversionista es «socialmente responsable» y elige adquirir instrumentos por una preferencia «ética». En este último caso puede enmarcarse la demanda de bonos verdes.

Son escasos y recientes los estudios que se han orientado a analizar si los inversionistas tienen una demanda diferenciada por instrumentos financieros amigables con el medio ambiente («instrumentos verdes») y por otros que no lo son.

Entre estos estudios, Reboredo, Quintela y Otero (2017) compararon el rendimiento de fondos mutuales asociados a proyectos de energía alternativa con el rendimiento de fondos mutuales convencionales. Para ello, estiman una variante del «alfa de Jensen» para cada caso. ${ }^{8}$ Su muestra abarca el período 2010-2016 y utilizan los rendimientos semanales observados en dichos fondos. Los autores consideraron portafolios cotizados tanto en dólares como en euros.

La ecuación considerada por Reboredo, Quintela y Otero (2017) es la siguiente:

$$
\alpha=\left(\mathrm{R}_{\mathrm{p}}-\mathrm{R}_{\mathrm{f}}\right)-\gamma_{1}\left(\mathrm{R}_{\mathrm{m}}-\mathrm{R}_{\mathrm{f}}\right)-\gamma_{2} \mathrm{SMB}-\gamma_{3} \mathrm{HML}-\gamma_{4} \text { MOMT }-\gamma_{5}\left(\mathrm{R}_{\mathrm{m}}-\mathrm{R}_{\mathrm{f}}\right)^{2}+\varepsilon
$$

Donde $R_{p}$ es el rendimiento del portafolio (ya sea de fondos mutuales de energía alternativa, ya sea de fondos mutuales convencionales), $R_{m}$ es el rendimiento del mercado, medido como el retorno del índice bursátil, $\mathrm{R}_{\mathrm{f}}$ es la tasa libre de riesgo (para los fondos en euros, la tasa Euribor; para los fondos en dólares, la tasa de bonos del Tesoro). La ecuación incorpora, además,

8 Jensen, M. (1968). El indicador «alfa» mide el rendimiento de un activo financiero en relación a un rendimiento teórico: $\alpha=R_{p}-\left[R_{f}+\beta\left(R_{m}-R_{f}\right)\right]$, donde $R_{p}$ es el rendimiento del portafolio, $R_{m}$ es el rendimiento del mercado, $R_{f}$ es la tasa libre de riesgo y $\beta$ es la $\beta$ del portafolio (volatilidad de este en relación con el mercado). Así, una alfa positiva sugiere que el portafolio tiene un rendimiento superior al rendimiento teórico. 
variables representativas de diversos riesgos: SMB representa el riesgo asociado al tamaño de la empresa, HML intenta capturar el riesgo de impago y MOMT es el riesgo de momento (medido como la diferencia entre el portafolio con los rendimientos más altos y el portafolio con los rendimientos más bajos en el período previo).

Para las alfas de los fondos mutuales de energía alternativa, los resultados arrojan no solo valores negativos, sino también valores inferiores a las alfas obtenidas para los fondos mutuales convencionales. De estos resultados, los autores concluyen que los inversionistas están dispuestos a pagar una prima por asignar sus fondos a proyectos de energía renovable: el rendimiento de los fondos mutuales de energía alternativa cotizados en euros es inferior en $13 \mathrm{pb}$ (puntos base) al de otros fondos mutuales, mientras que el rendimiento para el caso de fondos mutuales de energía alternativa cotizados en dólares es inferior en $26 \mathrm{pb}$.

A diferencia del trabajo de Reboredo, Quintela y Otero (2017), que se orienta a analizar fondos mutuales que cotizan diariamente, cuyo rendimiento se calcula por la variación en el precio del fondo y que incorpora variables adicionales que reflejan diversos riesgos (SMB, HML y MOMT), otros estudios se han enfocado en analizar específicamente la tasa a la que se colocan bonos verdes y compararla con la tasa a la que se colocan bonos convencionales, dado que los factores que explican sus rendimientos son prácticamente idénticos. Es decir, ambos tipos de bonos (verdes y convencionales) son emitidos en la misma moneda, institución, grado de inversión y duración.

Uno de estos estudios es el realizado por Zerbib (2016, 2019), quien utiliza un proceso de «emparejamiento» para bonos verdes y convencionales colocados por el mismo emisor. ${ }^{9}$ Así, para cada bono verde, Zerbib ubica los dos bonos convencionales con las fechas de vencimiento más cercanas colocados por el mismo emisor y con características iguales a las del bono verde: moneda, colateral y calificación de riesgo. Debido a que las fechas de vencimiento no son idénticas, el autor incorpora únicamente los bonos convencionales con fechas de vencimiento que no superen en dos años (ni antes ni después) la fecha de vencimiento del bono verde. Adicionalmente, tomando en consideración que puede haber una diferencia en la liquidez del bono verde y de su contraparte convencional - lo cual puede afectar su rendimiento- Zerbib incorpora dos limitantes adicionales: monto y fecha de

9 Este método, también conocido como «enfoque directo», consiste en emparejar dos instrumentos financieros con las mismas propiedades, excepto por la propiedad de interés en particular (en el caso de Zerbib, la característica verde de un bono). Este método fue implementado por Renneboog, Ter horst y Zhang (2008) para comparar el rendimiento de fondos de inversión calificados como «éticos» en relación con fondos convencionales. 
emisión. Así, restringe que los bonos convencionales i) tengan una fecha de emisión a lo más seis años después (o seis años antes) que la emisión del bono verde, y ii) que el monto de dicha emisión no sea superior a cuatro veces, ni inferior a una cuarta parte, del monto de la emisión del bono verde.

Zerbib obtiene datos de 110 bonos verdes cotizados en el mercado internacional y de los bonos convencionales considerados como contraparte. Los datos comprenden el período entre julio de 2013 y diciembre de 2017, y la ecuación estimada es la siguiente:

$$
\mathrm{p}_{\mathrm{i}}=\Delta \mathrm{y}_{\mathrm{i}, \mathrm{t}}-\beta \Delta \text { Liquidez }_{\mathrm{i}, \mathrm{t}}+\mathrm{e}_{\mathrm{i}, \mathrm{t}}
$$

Donde $\mathrm{p}_{\mathrm{i}}$ es el premio en el rendimiento del bono verde y $\Delta \mathrm{y}_{\mathrm{i}, \mathrm{t}}$ es la diferencia del rendimiento del bono verde en relación con el bono convencional $\left(\mathrm{y}^{\mathrm{V}}-\mathrm{y}^{\mathrm{C}}\right)$. Tomando en consideración que la liquidez puede incidir en el diferencial de rendimiento, Zerbib ajusta el premio $\left(\mathrm{p}_{\mathrm{i}}\right)$ por la diferencia en la liquidez entre el bono verde y el bono convencional ( $\Delta$ Liquidez). Esta última se estima por el margen de precio de compra respecto al precio de venta de cada bono (ask/bid spread). El resultado es que, en promedio, el bono verde tiene un ligero premio negativo (descuento) de 1.76 pb en relación con el bono convencional.

Ehlers y Packer (2017), por su parte, implementan una metodología de «emparejamiento» similar a la utilizada por Zerbib. A diferencia de este, incorporan como factor explicativo adicional que el bono verde haya sido certificado o verificado por un tercero. ${ }^{10}$ Estos autores corrigen también el amplio intervalo de tiempo en la fecha de emisión (+/- 6 años) considerado por Zerbib, y toman el bono convencional emitido en la fecha más próxima al bono verde. Así, comparan las tasas de colocación de 21 bonos verdes con las de bonos convencionales colocados por los mismos emisores. Al considerar únicamente la tasa a la que se coloca el bono y, por tanto, contar con un número limitado de observaciones, su análisis se enfoca únicamente en comparar estadísticas básicas de las tasas ofrecidas.

Los resultados de estos autores indican que no existe una diferencia en el rendimiento entre un bono verde y uno convencional cuando el primero no ha sido certificado por un tercero. No obstante, al considerar únicamente

10 No existe aún una definición internacionalmente aceptada de lo que significa «verde». Ello puede inducir mayores costos de búsqueda y de verificación a los inversionistas interesados en comprar instrumentos financieros «verdes». Una manera de mitigar estos costos de búsqueda es la certificación realizada por empresas calificadoras, las que verifican que los fondos obtenidos son asignados a proyectos de mejora ambiental. 
bonos verdes certificados, el resultado obtenido difiere: los bonos verdes tienen un rendimiento inferior, en promedio, en $18 \mathrm{pb}$ frente a los bonos convencionales. No obstante, los autores destacan que existe una variación considerable: la desviación estándar del premio es de $27 \mathrm{pb}$, mientras que cinco de los 21 bonos verdes analizados tienen un rendimiento superior al de los bonos convencionales.

Asian Bond Monitor (2018) realiza un ejercicio similar al de Zerbib (2016), es decir, «empareja» bonos verdes con bonos convencionales colocados por el mismo emisor, en la misma moneda y con la misma calificación crediticia. A diferencia de Zerbib, este estudio incorpora lo «verde» de un bono verde a través de una variable ficticia (dummy) que representa si el bono verde ha sido certificado por la Iniciativa para el Cambio Climático (CBI por sus siglas en inglés). ${ }^{11}$

Así, para una muestra de 60 emisiones de bonos verdes en el período 2010-2017, obtiene los rendimientos mensuales en el mercado secundario (tanto del bono verde como de su contraparte convencional) y estima la siguiente ecuación:

$$
\text { Premio }=\mathrm{a}_{0}+\mathrm{a}_{1} \text { Verde }+\mathrm{a}_{\mathrm{i}} \text { Control }_{\mathrm{i}}
$$

Donde «verde» corresponde a una variable ficticia que tiene el valor unitario si el bono verde cuenta con la certificación CBI; las variables de control también se incluyen como variables ficticias: para la calificación crediticia (AAA, AA, A y BBB), para la moneda de emisión (dólares, euros y no dólares/no euros), y sectoriales (financiero, servicios y otros).

El resultado de este estudio es que el rendimiento pagado por bonos verdes que no han sido certificados es igual que el de su contraparte convencional. No obstante, cuando los bonos verdes cuentan con la certificación del $\mathrm{CBI}$, su rendimiento es inferior, en promedio, en $7 \mathrm{pb}$ en relación con los convencionales. Ello, sugiere el artículo, destaca la importancia de la señal que otorga una agencia certificadora para reducir los costos de información de los inversionistas, quienes así están dispuestos a adquirir un bono verde con un rendimiento inferior.

Finalmente, es de destacar el trabajo de Östlund (2015), quien analiza 28 casos de bonos verdes y de sus contrapartes convencionales, obtenidas con la metodología de emparejamiento descrita anteriormente (mismo emisor,

11 Este es un esquema de certificación internacional que cuenta con esquemas de monitoreo y reporte para comprobar que los fondos obtenidos a través de un bono verde sean efectivamente asignados a financiar proyectos que mejoren el medio ambiente. 
moneda y riesgo). El número de casos es reducido toda vez que el autor, de 274 bonos verdes listados en la fecha considerada, descarta aquellos que i) no tuvieron una contraparte convencional, ii) tuvieron emisiones inferiores a diez millones de dólares (considerando que pueden tener problemas de liquidez) y iii) no presentaron una serie de precios continua.

El autor no realiza una estimación econométrica. En vez de ello, efectúa una prueba de hipótesis para aceptar (o no) que la diferencia en las medias del rendimiento de los bonos verdes $(\mathrm{Rg})$ y de los bonos convencionales $(\mathrm{Rc})$ es cero:

$$
\mathrm{H}_{0}: \mathrm{R}_{\mathrm{g}}-\mathrm{R}_{\mathrm{c}}=0
$$

Sus resultados indican que la hipótesis, al considerar la muestra total de bonos, no es rechazada. Así, a diferencia de los estudios previamente reseñados, Östlund concluye que no existe una preferencia por bonos verdes toda vez que no encuentra una diferencia estadísticamente significativa en sus rendimientos. Adicionalmente, el autor concluye que, al comprobar la hipótesis con pares de bonos individuales, 21 de los 27 casos rechaza $\mathrm{H}_{0}$ al encontrar un diferencial negativo en los rendimientos, mientras que los seis casos restantes rechaza $\mathrm{H}_{0}$ al encontrar un diferencial positivo.

\section{Análisis para el caso de México: metodología y resultados}

Como se mostró en la sección previa, el análisis comparativo entre bonos verdes y convencionales es aún muy reciente. Los resultados de los estudios referidos sugieren, en la mayoría de los casos, que hay una demanda diferenciada por ese tipo de bonos a nivel internacional, en particular cuando los bonos verdes cuentan con certificación. Asimismo, se destacó que los análisis utilizan información de los rendimientos observados (ya sea por la variación de precios, en el caso de fondos mutuales, o por el diferencial en el retorno en el mercado secundario de uno y otro tipo de bono). Estos estudios cuentan con datos de numerosas colocaciones de bonos a nivel internacional, los cuales se cotizan en el mercado secundario, lo que les permite tener series de tiempo suficientemente largas para realizar estimaciones econométricas y definir, a través de la comparación de los rendimientos y controlando por otras variables (moneda, plazo, riesgo, certificación, liquidez, etc.), si existen preferencias distintas entre un tipo de bono y otro.

En el caso del presente trabajo se presenta la limitante de que, como se mostró en la tabla 1, las emisiones de bonos verdes en el país han sido 
escasas (a octubre de 2018 solo se habían realizado ocho). Además, los inversionistas mantienen, por lo general, la tenencia de bonos verdes hasta el vencimiento, por lo que su comercialización en el mercado secundario es reducida. Ello limita considerablemente el número de datos disponibles para efectuar una estimación econométrica, como en la mayoría de los estudios referidos en la sección anterior. Dadas estas limitantes, para comprobar (o rechazar) la hipótesis de una demanda diferenciada por un bono verde y otro convencional, en este documento utilizamos las posturas de compra (ask price) de los demandantes de bonos durante el día en que se realiza la oferta de los mismos. De manera similar a los estudios previos, elegimos bonos (verdes y convencionales) emitidos por una misma institución: FEFA. ${ }^{12}$ Así, al comparar bonos colocados por el mismo emisor, denominados en la misma moneda (pesos) y con el mismo riesgo de crédito (calificación: AAA MEX por Fitch Ratings y mxAAA por Standard \& Poor's), la diferencia en las posturas de demanda, en caso de haberlas, reflejaría primordialmente una preferencia distinta entre un bono verde y un bono convencional. ${ }^{13}$

En el trabajo se presentan dos ejercicios para analizar si la demanda del bono verde tiene una distribución diferente que la de los convencionales. Para ello, se comparan inicialmente las posturas de demanda de los bonos FEFA ofrecidos en la misma fecha, 23 de octubre de 2018, tanto de los verdes (FEFA 18V, con plazo de vencimiento de 1092 días) como de los convencionales (FEFA 18-4, con plazo de vencimiento de 560 días, y FEFA $18-5$, con plazo de vencimiento de 1820 días). ${ }^{14}$ Dado que los montos ofrecidos fueron muy similares (2500 millones para el bono verde y 2875 millones

12 FEFA es el Fondo Especial para Financiamiento Agropecuario, que forma parte de FIRA (Fideicomisos Instituidos en Relación con la Agricultura). FEFA es el Fondo de FIRA que tiene atribuciones para colocar deuda en los mercados.

13 Es de reconocer que una limitante es controlar si el inversionista, en su manejo de portafolios, decide entre bonos FEFA u otro tipo de bonos. No obstante, cabe mencionar que el día de la emisión de los bonos de FEFA (23 de octubre de 2018) no hubo colocaciones de papel corporativo o del gobierno. El 18 de octubre se realizaron las siguientes colocaciones de deuda corporativa: Grupo Famsa (a 196 días), Mercader Financial (SOFOM, a 308 días), Navistar Financial (SOFOM, a 252 días), Finactiv (SOFOM, a 364 días) y Arrendadora Actinver (a 28 días); el 25 de octubre se colocó papel de Aeroméxico (a 168 días). Dado el menor plazo y el mayor riesgo de estos instrumentos en relación con los bonos FEFA, podría concluirse que el papel corporativo no afectó en gran medida la demanda de bonos FEFA.

14 Se toman en cuenta estos dos bonos dado que fueron colocados mediante «vasos comunicantes». 
para los bonos convencionales), esta comparación también limita el posible problema de liquidez mencionado en los estudios previos. ${ }^{15}$

El segundo ejercicio toma en cuenta que la diferencia en el plazo de vencimiento de los bonos puede afectar sus respectivas posturas de demanda. Así, se comparan las posturas de demanda del bono verde del 23 de octubre (plazo de vencimiento de 1092 días) con las de los convencionales que tienen el mismo plazo de vencimiento (1092 días), realizadas en las fechas previas más próximas (FEFA 18-2, en mayo de 2018, y FEFA 17-2, en mayo de 2017). Esta elección cumple con el método de emparejamiento sugerido por Zerbib, toda vez que la diferencia en la fecha de colocación (5 y 17 meses) es considerablemente menor a la considerada por el autor (6 años).

La información utilizada para ambos ejercicios proviene del «libro ciego» de cada una de las colocaciones, que reporta, para cada postura de demanda, la hora, el monto y tasa/precio solicitado, así como el monto y tasa/ precio asignado. Adicionalmente, es de destacar que el bono verde de FEFA fue certificado por la empresa Sustainalytics, lo cual facilita la identificación de tal característica a los inversionistas demandantes de este tipo de instrumentos, en línea con los trabajos del Asian Bond Monitor (2018) y de Ehlers y Packer (2017).

En ambos ejercicios se realiza una prueba $\chi^{2}$ para identificar si la distribución de las posturas de demanda para uno y otro tipo de bono provienen de una distribución diferente. El resultado es relevante: sí es posible encontrar una diferenciación en la distribución de las posturas de los inversionistas para adquirir un bono verde respecto a uno convencional, los emisores de los primeros no tienen necesariamente que ofrecer incentivos para colocar su papel o, alternativamente, los costos asociados a una certificación de un bono verde pueden ser absorbidos con una menor tasa del mismo; los inversionistas reflejan sus preferencias ambientales, no pecuniarias, aceptando un menor rendimiento.

En la siguiente tabla se presentan las principales características de los bonos referidos: montos (convocado, solicitado y asignado, cifras en millones de pesos), plazo de vencimiento (días) y tasas mínimas, máximas y asignadas (puntos porcentuales adicionales a TIIE).

15 Como se destacó en la sección anterior, para poder comparar un bono verde con uno convencional, Zerbib considera que el monto de emisión del bono verde no sea ni mayor a cuatro veces ni menor al $25 \%$ de la emisión del bono convencional. 
Tabla 2. Colocaciones FEFA. Datos generales

\begin{tabular}{|l|c|c|c|c|c|}
\hline & \multicolumn{3}{|c|}{ Colocación 23 de octubre } & \multicolumn{2}{c|}{ Mismo plazo } \\
\hline & $\begin{array}{c}\text { FEFA 18V } \\
\text { (verde) }\end{array}$ & $\begin{array}{c}\text { FEFA } \\
\mathbf{1 8 - 5}\end{array}$ & $\begin{array}{c}\text { FEFA } \\
\mathbf{1 8 - 4}\end{array}$ & $\begin{array}{c}\text { FEFA } \\
\mathbf{1 8 - 2}\end{array}$ & $\begin{array}{c}\text { FEFA } \\
\mathbf{1 7 - 2}\end{array}$ \\
\hline Monto convocado & 2500 & 2875 & 2875 & 5750 & 3000 \\
\hline Monto solicitado & 4587.43 & 1237.14 & 4694 & 6642 & 6471 \\
\hline Monto asignado & 2500 & 610.66 & 2264 & 2300 & 2300 \\
\hline Plazo (días) & 1092 & 1820 & 560 & 1092 & 1092 \\
\hline Sobredemanda & 1.83 & 0.43 & 1.63 & 1.16 & 2.16 \\
\hline $\begin{array}{l}\text { Tasa mínima } \\
\text { solicitada }\end{array}$ & 0.05 & 0.19 & 0 & 0.1 & 0.2 \\
\hline $\begin{array}{l}\text { Tasa máxima } \\
\text { solicitada }\end{array}$ & 0.28 & 0.39 & 0.15 & 0.33 & 0.45 \\
\hline $\begin{array}{l}\text { Tasa única } \\
\text { asignada }\end{array}$ & 0.15 & 0.25 & 0.1 & 0.2 & 0.35 \\
\hline
\end{tabular}

Fuente: elaboración propia con base en datos de la Bolsa Mexicana de Valores. Los montos se presentan en millones de pesos. Las tasas se refieren a puntos porcentuales sobre TIIE. El mismo plazo se refiere a colocaciones a 1092 días, como fue la de FEFA 18V. La sobredemanda se mide por la relación entre el monto solicitado y el monto asignado.

En la primera parte del cuadro se presenta la comparación del bono verde FEFA $18 \mathrm{~V}$ con los convencionales FEFA 18-4 y FEFA 18-5, todos ellos emitidos en la misma fecha (23 de octubre de 2018). Si bien existe el pormenor de que el plazo de vencimiento no es el mismo, ello se mitiga de alguna manera al considerar que la emisión de los bonos convencionales se efectúa a través de vasos comunicantes (una colocación a 560 días, la otra a 1820 días). Esta situación explica que las posturas de demanda de los bonos convencionales se dirigiera primordialmente hacia aquellos de menor plazo de vencimiento (560 días, mismos que tuvieron una demanda de 1.63 veces el monto convocado), respecto a los bonos convencionales con mayor plazo de vencimiento (1820 días, que tuvieron una relación de demanda a oferta de únicamente 0.43 veces). Es de resaltar también que la sobredemanda de bonos verdes (1.83 veces el monto convocado), a pesar de tener un plazo de 1092 días, haya sido superior a cualquiera de los bono convencionales.

La distribución de las posturas de demanda se presenta en las siguientes gráficas, ordenadas por rendimiento pagado (sobretasa respecto a TIIE). 
En el caso de los bonos convencionales, dado que se colocaron a través de «vasos comunicantes», se sumaron las posturas respectivas. En una primera comparación gráfica, es factible sugerir que la demanda de los inversionistas difiere entre el bono verde y los bonos convencionales. ${ }^{16}$ Más adelante se realizan pruebas estadísticas tanto para comprobar o rechazar dicha hipótesis como para analizar si la diferencia en las tasas pagadas es o no cero.

Gráficas 1 y 2. Comparativo del bono verde con otros bonos (mismo día de emisión, diferente plazo de vencimiento)

Gráfica 1. FEFA bono verde (distribución y sobretasa)

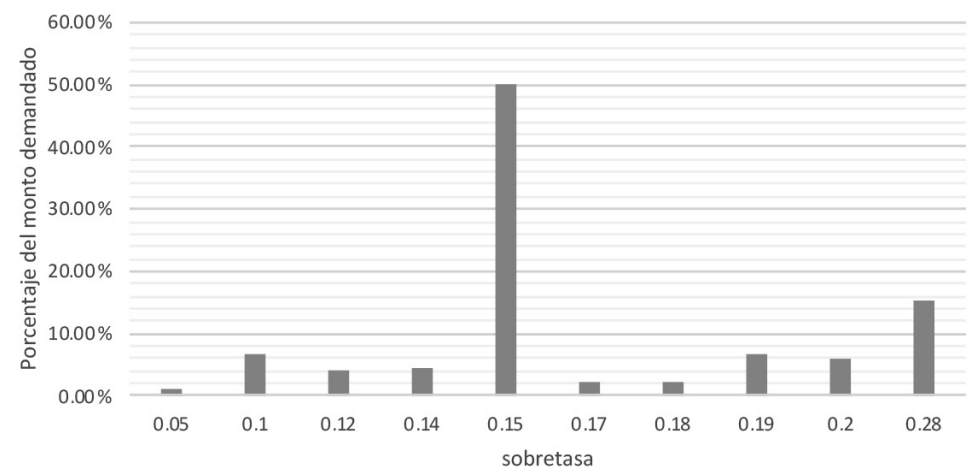

Fuente: Bolsa Mexicana de Valores.

Gráfica 2. FEFA bonos 18-4 y 18-5 (distribución y sobretasa)

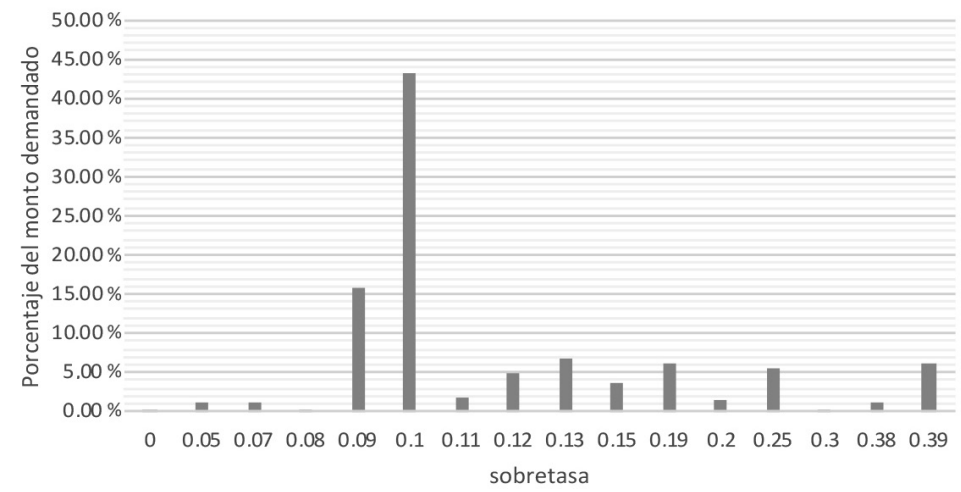

Fuente: Bolsa Mexicana de Valores. Bonos colocados a través de vasos comunicantes.

16 La moda en el primero de ellos fue TIIE $+15 \mathrm{pb}$; en el caso de los segundos fue TIIE $+10 \mathrm{pb}$. 
Un segundo ejercicio consiste en comparar el bono verde con bonos convencionales colocados con el mismo plazo de vencimiento (1052 días), con el objetivo de evitar el efecto que colocaciones con plazos de vencimiento diferentes puede tener en la tasa demandada en las colocaciones. En este caso, los datos incorporados en la segunda parte de la tabla 2 indican que, tanto la tasa asignada como las tasas mínimas y máximas demandadas fueron inferiores en el bono verde que en los bonos convencionales.

Gráficas 3, 4 y 5. Comparativo del bono verde con otros bonos (mismo plazo de vencimiento, diferente día de emisión)

Gráfica 3. FEFA Bono verde (distribución y sobretasa)

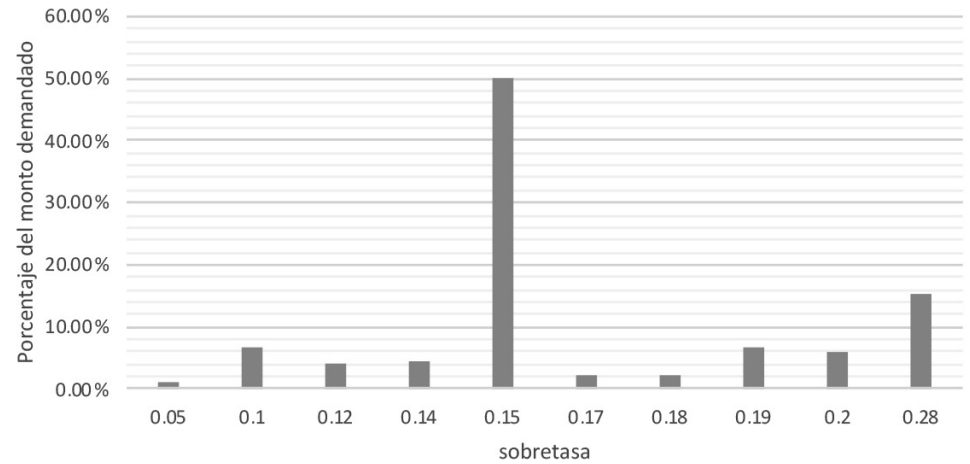

Fuente: Bolsa Mexicana de Valores.

Gráfica 4. FEFA Bono 18-2 (Distribución y sobretasa)

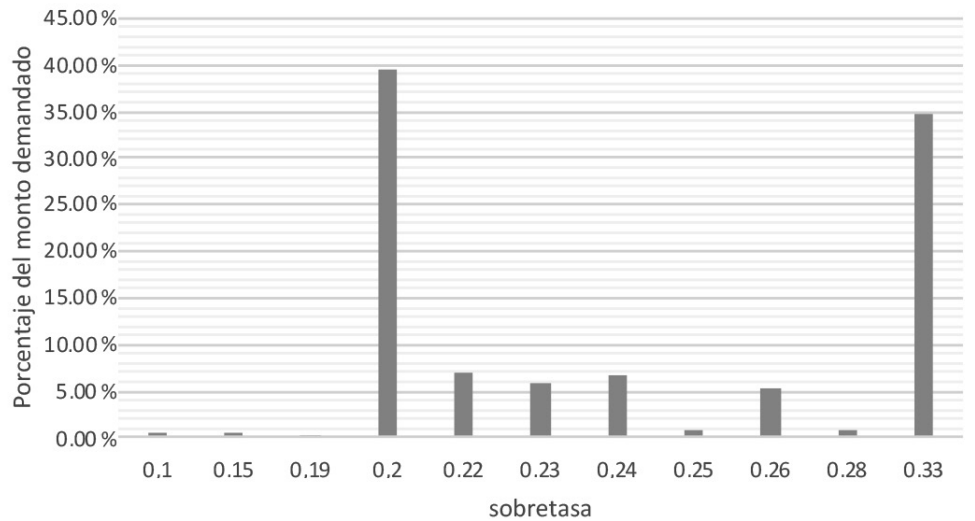

Fuente: Bolsa Mexicana de Valores. 
Gráfica 5. FEFA Bono 17-2 (Distribución y sobretasa)

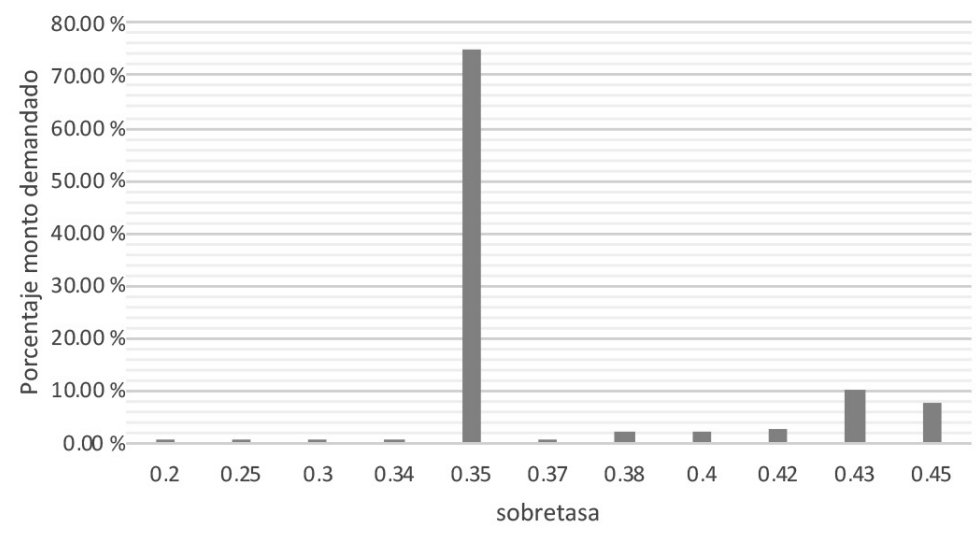

Fuente: Bolsa Mexicana de Valores.

Para formalizar el análisis y determinar si las posturas de demanda realizadas por los inversionistas para adquirir el bono verde pueden considerarse similares a las de bonos convencionales, se efectúa la prueba estadística $\chi^{2}$ que permite identificar si dichas posturas provienen de la misma distribución o si pueden considerarse independientes. En caso de que provengan de la misma distribución, puede inferirse que no hay una demanda diferenciada por el bono verde en relación con el bono convencional. Por el contrario, si las distribuciones son independientes, los resultados sugerirían que sí hay una demanda diferenciada entre dichos bonos.

La prueba estadística se especifica como se muestra a continuación:

$$
\chi^{2}=\sum i \sum j \frac{(n i j-E i j)^{2}}{E i j}
$$

Para realizarla, se construye una matriz de frecuencias, donde los renglones son las sobretasas ofertadas por los inversionistas (en relación con el TIIE), y las columnas son el número de posturas para cada tipo de bono (verde o convencional). ${ }^{17}$ Así, nij es la observación de la sobretasa i ofrecida para las posturas del bono tipo $\mathrm{j}$.

El número total de posturas para una determinada sobretasa es:

$$
R j=\Sigma n i j
$$

17 El total de posturas para ambos tipos de bono emitidos en la misma fecha fue 85; el total de posturas para ambos tipos de bono emitidos con el mismo plazo fue 64 . 
El número total de posturas por tipo de bono es:

$$
C_{\mathrm{i}}=\Sigma n i j
$$

Y la frecuencia de cada una de las posturas:

$$
E i j=(R i-C j) / N
$$

En la prueba, $\mathrm{H}_{\mathrm{o}}$ es la hipótesis de que no existen diferencias significativas entre los elementos de las muestras y $\mathrm{H}_{1}$ de que sí existen tales diferencias.

La regla de decisión es la siguiente:

- si $\chi^{2} \leq \chi_{\mathrm{T}}^{2}, \mathrm{H}_{\mathrm{o}}$ no puede ser rechazada, es decir, no existe diferencia en la distribución de las posturas de demanda para uno y otro tipo de bono, y puede concluirse que la demanda no es diferenciada;

- si $\chi^{2}>\chi^{2}{ }_{\mathrm{T}} \mathrm{H}_{\mathrm{o}}$ debe ser rechazada, por lo que puede concluirse que la distribución de las posturas de demanda se refleja en distribuciones independientes y que la demanda por uno y otro tipo de bono es diferenciada.

El resultado de la prueba se muestra en la tabla 3.

Tabla 3. Resultados de las pruebas*

\begin{tabular}{|l|c|c|}
\hline & $\chi^{2}$ obtenida & $\chi^{2}$ de tablas \\
\hline Bonos verdes respecto a colocaciones mismo día & 69.25 & 36.19 \\
\hline $\begin{array}{l}\text { Bonos verdes respecto a colocación mismo plazo } \\
\text { FEFA 18-2 }\end{array}$ & 92.5 & 21.66 \\
\hline $\begin{array}{l}\text { Bonos verdes respecto a colocación mismo plazo } \\
\text { FEFA 17-2 }\end{array}$ & 91.14 & 23.21 \\
\hline
\end{tabular}

*19 grados de libertad para posturas de bonos el mismo día (FEFA 18V y FEFA 18-4 y 18-5); nueve y diez grados de libertad para las posturas de bonos verdes (FEFA 18V) en relación con FEFA 18-2 y FEFA 17-2 respectivamente. El valor de la $\mathrm{X}^{2}$ de las tablas es al $99 \%$ de confianza, por lo que puede considerarse que las pruebas son robustas.

Fuente: elaboración propia.

Como se observa en la tabla 3 , tanto al comparar las posturas de los inversionistas para bonos verdes con las de convencionales en el mismo día (23 de octubre de 2018), como al compararlas para bonos verdes y convencionales para los mismos plazos (FEFA 18-2 y FEFA 17-2, todas a 1052 días) la $\chi^{2}$ obtenida es mayor que la de tablas $\left(\chi^{2}>\chi^{2}\right.$ T), por lo 
que la hipótesis nula (no hay diferencias significativas en la distribución de las posturas de los inversionistas para los diferentes tipos de bonos) es rechazada al $99 \%$ de confianza.

Si bien estos resultados sugieren que las posturas de demanda para bonos verdes provienen de una distinta distribución que para convencionales, ello no implica necesariamente que haya una diferencia en las tasas asignadas para uno u otro tipo de bono, como es la conclusión de la mayoría de los estudios referidos en el marco teórico. Para ello, siguiendo el estudio de Östlund (2015), analizamos si la diferencia en las tasas demandadas es o no cero, formulando las siguientes hipótesis:

$$
\begin{aligned}
& \mathrm{H}_{0} \text { : tasa del bono verde }- \text { tasa del bono convencional }=0 \\
& \mathrm{H}_{1} \text { : tasa del bono verde }- \text { tasa del bono convencional } \neq 0
\end{aligned}
$$

Así, en caso de rechazar $\mathrm{H}_{0}$, podría inferirse que no solo la distribución de las posturas de demanda es diferente (como se presentó en el apartado anterior) sino que ello también se refleja en un diferencial en las tasas asignadas. En particular, en línea con los resultados de la mayoría de los estudios reseñados previamente, se intentaría comprobar que el diferencial de tasas es negativo, es decir, que al presentar sus posturas de demanda los inversionistas de bonos verdes están dispuestos a aceptar una tasa de interés inferior a la de los bonos convencionales, sugiriendo que su demanda por bonos verdes tiene un componente no pecuniario.

Para comprobar lo anterior, se analiza la diferencia en las medias de las tasas para una y otra postura de demanda y se construye un intervalo de confianza para cada caso: 18

$$
\mathrm{RV}-\mathrm{Rc} \pm_{\mathrm{t}(95 \% / 2, \mathrm{n}+\mathrm{m}-2)} \mathrm{S}_{\mathrm{p}} \sqrt{ }(1 / \mathrm{n})+(1 / \mathrm{m})
$$

Donde $\mathrm{Rv}$ es la tasa promedio demandada para el bono verde, Rc la tasa promedio demandada para el bono convencional, $\mathrm{n}$ y $\mathrm{m}$ son el número de casos (grupos) de posturas de demanda para el bono verde y el convencional, respectivamente, y $\mathrm{s}_{\mathrm{p}}$ es la desviación estándar de las dos muestras. ${ }^{19}$ Los principales datos utilizados se presentan en la tabla 4.

18 Ross (2014: 253-260).

$\left.19 \mathrm{~S}_{\mathrm{p}}{ }_{\mathrm{p}}=\left((\mathrm{n}-1) \mathrm{S}_{1}{ }_{1}+(\mathrm{m}-1) \mathrm{S}_{2}{ }_{2}\right) /(\mathrm{n}+\mathrm{m}-2)\right)$, donde $\mathrm{S}_{1}{ }_{1}$ es la varianza de las posturas de demanda del bono verde y $\mathrm{S}_{2}{ }_{2}$ es la varianza de las posturas de demanda del bono convencional. Al $95 \%$ de confianza la t de tablas con 23 grados de libertad (caso de bonos emitidos en la misma fecha) es 2.069; su valor con 17 grados de libertad (caso de bonos con el mismo plazo) es 2.11 . 
Tabla 4. Estadísticas generales de posturas de demanda de bonos FEFA

\begin{tabular}{|l|c|c|c|c|}
\hline & \multicolumn{2}{|c|}{ Colocación $\mathbf{2 3}$ de octubre } & \multicolumn{2}{|c|}{ Mismo plazo } \\
\hline & $\begin{array}{c}\text { Bono } \\
\text { verde }\end{array}$ & $\begin{array}{c}\text { Bono } \\
\text { convencional }\end{array}$ & $\begin{array}{c}\text { Bono } \\
\text { conv. 1 }\end{array}$ & $\begin{array}{c}\text { Bono } \\
\text { conv. 2 }\end{array}$ \\
\hline $\begin{array}{l}\text { Tasa promedio } \\
\text { demandada }\end{array}$ & 0.158 & 0.174 & 0.223 & 0.354 \\
\hline Desviación estándar & 0.0625 & 0.1098 & 0.063 & 0.08 \\
\hline Casos & 10 & 15 & 11 & 11 \\
\hline
\end{tabular}

Fuente: elaboración propia con base en información de las colocaciones de FEFA, Bolsa Mexicana de Valores.

Los intervalos obtenidos al $95 \%$ de confianza son:

a. Misma fecha de colocación

Tasa bono verde - tasa bono convencional $(-0.0955,0.0635)$

b. Mismo plazo

Tasa bono verde - tasa bono convencional1 $(-0.1257,-0.0037)$

Tasa bono verde - tasa bono convencional $2(-0.2646,-0.1266)$

En el primer caso - misma fecha de colocación ( 23 de octubre) - el intervalo de confianza incorpora el valor cero, por lo que no es posible rechazar la hipótesis nula. Así, a pesar de que las distribuciones en las posturas de demanda son diferentes para el bono verde y los bonos convencionales colocados en la misma fecha, no hay una diferencia estadísticamente significativa en la media de las tasas demandadas.

Por el contrario, en el segundo caso, en el que se analizan las posturas de demanda para el bono verde y los dos bonos convencionales emitidos con el mismo plazo de vencimiento (1052 días), el intervalo de confianza no incluye el valor cero. Por ello, es posible rechazar la hipótesis nula y puede concluirse que sí hay una diferencia — en este caso negativa — estadísticamente significativa: la media de las tasas demandadas por el bono verde es inferior a la media de las tasas demandadas para cualquiera de los bonos convencionales. Ello sugiere que, en este caso, los inversionistas sí presentan motivos no pecuniarios para demandar bonos verdes, aceptando un menor rendimiento. ${ }^{20}$

$20 \mathrm{Al}$ intentar identificar si existían factores de riesgo no incorporados, se revisó el valor del índice de bonos para mercados emergentes (EMBI, Emerging Market Bond Index) para México. Tanto en octubre de 2018 como en mayo del mismo año y en el mismo mes de 2017 (meses en que se emitieron los bonos que se comparan), el indicador de riesgo osciló entre 190 y 200 puntos. Ello sugiere que las 
En suma, el análisis presentado en esta sección sugiere que las posturas de demanda (ask prices) de bonos verdes sí tiene una distribución diferente a la de bonos convencionales. Ello ocurre tanto para la comparación de bonos emitidos en la misma fecha como cuando se compararon bonos con el mismo plazo de vencimiento. Sin embargo, únicamente en el segundo caso es factible concluir que los inversionistas están dispuestos a aceptar una menor tasa al adquirir bonos verdes.

\section{Conclusión}

El sistema financiero amigable con el medio ambiente ha adquirido cada vez mayor importancia como vía para enfrentar el cambio climático y otros riesgos ambientales. En México, diversas instituciones han impulsado ese objetivo. Para lograrlo, por ejemplo, la Bolsa Mexicana de Valores estableció el Índice de Precios y Cotizaciones Sustentable y creó la organización $\mathrm{MexiCO}_{2}$. Asimismo, la mayoría de las grandes instituciones financieras implementan, para la medición y control de riesgos ambientales, el Sistema de Administración de Riesgos Ambientales y Sociales (SARAS). Finalmente, cabe destacar que la Asociación de Bancos de México desarrolló el «Protocolo de Sustentabilidad», al que se han afiliado numerosas instituciones financieras. En este entorno, los bonos verdes constituyen un instrumento relevante para financiar proyectos ambientalmente amigables y, así, desarrollar el mercado verde.

Durante la presente década, la colocación de bonos verdes se ha desarrollado rápidamente a nivel internacional. No obstante, las emisiones de estos bonos han sido escasas en nuestro país. El presente documento analiza si, derivada de razones no pecuniarias, existe en México una demanda diferenciada entre el bono verde y el convencional. De no haberla, podría dificultarse el desarrollo del mercado verde, toda vez que los inversionistas podrían asignar sus recursos hacia bonos convencionales.

A diferencia de estudios previos que se orientan a comparar los rendimientos observados de bonos verdes y convencionales en el mercado secundario, en este trabajo recurrimos a la información de las posturas de demanda (ask price) realizadas por inversionistas el día de la colocación. Ello debido no solo a que las colocaciones de bonos verdes en nuestro país han sido escasas, sino también a que la mayoría de los inversionistas los

condiciones de riesgo eran muy similares, por lo que el diferencial en tasas puede ser explicable por las diferentes preferencias de los inversionistas. 
mantiene a vencimiento, por lo que las transacciones en el mercado secundario son limitadas.

Los bonos elegidos - tanto verdes como convencionales - son los que emite el Fondo Especial para Financiamiento Agropecuario (FEFA), calificados por Fitch Ratings y Standard \& Poor's con alto grado de inversión, emitidos en la misma moneda y con montos relativamente similares. Ello implicaría un nivel de riesgo cercano para ambos tipos de bono. Las posturas de demanda se comparan para bonos colocados, por tanto, el mismo día, aunque con plazos de vencimiento diferentes, así como en días diferentes, con el mismo plazo de vencimiento.

En el trabajo se realizan dos análisis. El primero, para comprobar si existe una demanda diferenciada para uno y otro tipo de bono. Con ese objetivo, se analiza la distribución de las posturas de demanda en uno y otro caso. Los resultados sugieren que las demandas de los inversionistas, ante un papel emitido por la misma institución, son diferenciadas en el caso de un papel verde y otro convencional, independientemente de la fecha de emisión y de vencimiento.

En el segundo análisis se intenta corroborar si existe una diferencia en las medias del rendimiento (ask price) en las posturas de demanda del bono verde y de los convencionales. Ello se comprueba únicamente cuando dichos bonos se emitieron con el mismo plazo de vencimiento. En este caso, los inversionistas están dispuestos a aceptar rendimientos inferiores en los bonos verdes en relación con los convencionales. Es decir, un bono verde puede pagar rendimientos ligeramente inferiores pues hay una demanda «ambientalista» por el mismo. Este resultado está en línea con los obtenidos en los estudios de Asian Bond Monitor (2018), Ehlers y Packer (2017) y (Zerbib 2016, 2019). En contraparte, cuando se compara el bono verde con el convencional con la misma fecha de emisión, la prueba de hipótesis realizada no permite llegar a la misma conclusión. Este último punto concuerda con los resultados de Östlund (2015).

Así, si bien la distribución de las posturas de demanda es diferente para un bono verde y uno convencional, la media de sus respectivos rendimientos no siempre es distinta. Cabe mencionar que el resultado del estudio tiene la limitante de tomar datos de las posturas de demanda realizadas en una sola colocación de bono verde (FEFA 18V). Una veta para estudios posteriores consiste en profundizar el estudio una vez que se incremente en el país el número de colocaciones de bonos verdes e intentar utilizar datos de las colocaciones de bonos verdes y convencionales de otros bancos de desarrollo. 


\section{Los autores}

Lucía Pérez Delgado es licenciada en Economía por el ITAM; tiene una maestría en Economía por la Universidad de California en Los Ángeles y un doctorado en Ciencias Sociales y Políticas por la Universidad Iberoamericana (UIA). Ha sido coordinadora de la carrera de economía en la Universidad Anáhuac del Norte y Directora de Vinculación y Educación Continua en el CIDE. Actualmente imparte cursos en la Facultad de Economía y en la Facultad de Medicina de la UAN, y en la UIA. Sus intereses particulares son economía monetaria, economía de la salud y economía ambiental.

Correo electrónico: arellanolucia@hotmail.com

Rogelio Arellano Cadena es licenciado en Economía por el ITAM y tiene una maestría y doctorado en Economía por la Universidad de California en Los Ángeles. Fue ministro-representante de la Secretaría de Comercio ante la OCDE y consultor para el Banco Interamericano de Desarrollo y el Centro de Estudios Monetarios Latinoamericanos. Actualmente es investigador en el Banco de México, en donde se desempeñó como Gerente de Fideicomisos de Fomento. Es profesor de asignatura en la UAN. Sus intereses son economía monetaria y economía ambiental.

Correo electrónico: arellanorogelio61@hotmail.com 
Referencias bibliográficas

Asian Bond Monitor (junio de 2018). «The role of greenness indicators in green bond market development: an empirical analysis». Asian Development Bank: 40-51.

Banco Mundial (2018). Groundswell. Preparing for Internal Climate Migration. Recuperado de: https://openknowledge.worldbank.org/ handle/10986/29461

Banco Mundial, International Finance Corporation (2016). ClimateSmart Investment Potential in Latin America: A Trillion Dólar Opportunity. Washington, Banco Mundial. Recuperado de: https://www. ifc.org/wps/wcm/connect/0d9f8fbf-2738-4432-843c-05184b9546d8/ LAC+1Trillion+6-13-16+web+FINAL.pdf?MOD=AJPERES

Cheng, B., Ioannou, I. y Serafeim, G. (2014): «Corporate social responsibility and access to Finance». Strategic Management Journal, vol. 35, núm. 1: 1-23. Doi: $10.1002 / \mathrm{smj} .2131$

Comisión Europea (2016). Study on the potential of green bond finance for resource-efficient investments. Luxemburgo, Unión Europea. Doi: $10.2779 / 234777$

Consejo Consultivo de Finanzas Climáticas, CCFC (2016). Principios de Bonos Verdes MX. México, Bolsa Mexicana de Valores. Recuperado de: https://www.bmv.com.mx/docs-pub/MI_EMPRESA_EN_BOLSA/ CTEN_MINGE/PRIN_BONOS_VERDES_MX2_1.pdf

Ehlers, T. y Packer, F. (2017). «Green bond Finance and certification». BIS Quarterly Review: 89-103. Recuperado de: https://www.bis.org/publ/ qtrpdf/r_qt1709h.pdf

Fama, E. y French, K. (2007). «Disagreement, tastes, and asset prices». Journal of Financial Economics 83: 667-689.

Federal Ministry for Economic Cooperation and Development (2018). Green Bonds: Ecosystem, Issuance Process and Regional Perspectives. Mexico Edition. Bonn, Eschborn, Alemania: (GIZ) GmbH. Recuperado de: http://www.emergingmarketsdialogue.org/wp-content/uploads/2018/06/ GIZ-SEB-ABM_Green-Bonds_Mexico-Edition.pdf

Foro Económico Mundial (2018). Informe de riesgos mundiales $2018\left(13^{\mathrm{a}}\right.$ ed.). Ginebra: World Economic Forum. Recuperado de https://www. $\mathrm{mmc} . \mathrm{com} / \mathrm{content} / \mathrm{dam} / \mathrm{mmc}-\mathrm{web} /$ Global-Risk-Center/Files/the-globalrisks-report-2018-es.pdf

Grupo Intergubernamental de Expertos sobre Cambio Climático (2013). Cambio Climático 2013. Bases Físicas. (s/1). OMM, PNUMA. Recuperado de: https://www.ipcc.ch/pdf/assessment-report/ar5/wg1/WG1AR5 SummaryVolume_FINAL_SPANISH.pdf

Instituto Nacional de Ecología y Cambio Climático, INECC (2014). Actualización y divulgación de los nuevos escenarios de cambio climático aplicados a México para fortalecer las capacidades nacionales. México, INECC. Recuperado de: https://www.gob.mx/inecc/documentos/actualizacion- 
y-divulgacion-de-los-nuevos-escenarios-de-cambio-climatico-aplicados-amexico-para-fortalecer-las-capacidades-nacionales

International Capital Market Association, ICMA (2018). "Los Principios de los Bonos Verdes 2018 - Green Bond Principles (GBP) Guía del Procedimiento Voluntario para la Emisión de Bonos Verdes". Recuperado de: https://www.icmagroup.org/green-social-and-sustainability-bonds/ green-bond-principles-gbp/

Jensen, M. (1968). «The performance of mutual funds in the period 19451964». Journal of Finance, vol. 23: 389-416. Doi: 10.2139/ssrn.244153

Los principios del Ecuador (2013). Recuperado de http://equator-principles. com/wp-content/uploads/2018/01/equator_principles_spanish_2013.pdf

$\mathrm{MexiCO}_{2}$ (2018). «Bonos etiquetados emitidos en México». México, Mexi$\mathrm{CO}_{2}$ (sitio de internet). Recuperado de: http://www.mexico2.com.mx/ uploadsmexico/file/Bonos\%20Emitidos\%20en\%20México(2).pdf

$\mathrm{MexiCO}_{2}$ (19 de marzo de 2017). Bonos verdes. «Los bonos verdes como alternativa de financiamiento para los proyectos de infraestuctura». Tercer Seminario de Asociación Público-Privada de México: Impulso al Desarrollo de Infraestructura para la Sociedad. Ciudad de México. Recuperado de: https://www.gob.mx/cms/uploads/attachment/file/208275/ Presentacion_BV-APPs_Alba_Aguilar.pdf

Moid, S. (2018). «Green bonds: country experiences, challenges and opportunities». Rajagiri Management Journal, vol.11: 63-78. Recuperado de: http://journals.rajagiri.edu/index.php/rmj/article/view/16

Nieto, M. (2017). Banks and environmental sustainability: some financial stability reflections (Documento de trabajo). International Research Centre on Cooperative Finance.

Organización para la Cooperación y el Desarrollo Económico, OCDE (2017). Investing in Climate, Investing in Growth. Recuperado de: https://read.oecd-ilibrary.org/economics/investing-in-climate-investingin-growth_9789264273528-en\#page1

Organización para la Cooperación y el Desarrollo Económico, OCDE/Asociación Internacional de Mercados de Capital (2016). Green Bonds: Country Experiences, Barriers and Options. (s.p.i.) Recuperado de: http:// unepinquiry.org/wp-content/uploads/2016/09/6_Green_Bonds_Country_ Experiences_Barriers_and_Options.pdf

Östlund, E. (2015). «Are investors rational profit maximisers or do they exhibit a green preference? Evidence from the green bond market». Stockholm School of Economics Department of Economics, tesis de maestría en Economía, 2014-2015. Recuperado de http://arc.hhs.se/ download.aspx?MediumId=2494

Pérez Delgado, L. y Arellano, R. (2018). Enverdecimiento del Sector Financiero. México: Instituto de Desarrollo Universidad Anáhuac, Actualización 70.

Programa de las Naciones Unidas para el Desarrollo, PNUD (2011). Catalizando el financiamiento para enfrentar el cambio climático. Nueva York: 
PNUD. Recuperado de: http://www.undp.org/content/undp/es/home/ librarypage/environment-energy/catalyzing-climate-finance.html

Programa de las Naciones Unidas para el Medio Ambiente, PNUMA (2018). Making Waves. Aligning the Financial System with Sustainable Development. Ginebra: PNUMA. Recuperado de: http://unepinquiry.org/ wp-content/uploads/2018/04/Making_Waves_lowres.pdf

Reboredo, Juan C., Quintela, M. y Otero, L. (2017). «Do investors pay a Premium for going green? Evidence form alternative energy mutual funds». Renewable and Sustainable Energy Reviews, vol. 73: 512-520.

Renneboog, L., Ter Horst, J. y Zhang, C. (2008). «The price of ethics and stakeholder governance: the performance of socially responsible mutual funds». Journal of Corporate Finance: 302-322.

Ross, S. (2014). Introduction to Probability and Statistics for Engineers and Scientists ( $3^{\mathrm{a}}$ ed.). San Francisco: Elsevier.

Sustainable Banking Network, World Bank International Finance Corporation (2018). Creating green bond markets. Insights, Innovations, and Tools from Emerging Markets (en línea). Recuperado de: https://www.ifc. org/wps/wcm/connect/2448e622-3e36-4098-b228-ffe52185af51/SBN+ Creating+Green+Bond+Markets+Report+Toolkit.pdf?MOD=AJPERES

Task Force on Climate Related Financial Disclosures (2017). Final Report. Recommendations of the Task Force on Climate-related Financial Disclosures (en línea). Recuperado de: https://www.fsb-tcfd.org/publications/ final-recommendations-report/

Zerbib. O.D. (2016). «Is There a Green Bond Premium? The Yield Differential Between Green and Conventional Bonds». Recuperado de: https:// papers.ssrn.com/sol3/papers.cfm?abstract id $=2889690$

Zerbib, O.D. (2019): «The effect of pro-environmental preferences on bond prices: Evidence from green bonds». Journal of Banking and Finance, 98: 39-60. 


\section{LINEAMIENTOS PARA LOS AUTORES \\ The Anáhuac Journal: Business and Economics es una revista semestral de la Facultad de Economía y Negocios de \\ la Universidad Anáhuac México.}

The Anáhuac Journal recibe artículos inéditos que no se hayan publicado en ningún medio impreso o electrónico, ni que hayan sido postulados de forma simultánea para su publicación en otras revistas u órganos editoriales.

Los artículos deben ser producto de investigaciones y estudios con resultados originales en las siguientes líneas temáticas:
a) Derecho corporativo
g) Mercadotecnia
b) Gobierno corporativo
h) Economía y estrategia
c) Responsabilidad social empresarial
i) Finanzas y contaduría
d) Ética empresarial
j) Toma de decisiones
e) Liderazgo y dirección
k) Economía
f) Emprendimiento e innovación
1) Innovación

\section{Sobre el proceso}

1. The Anáhuac Journal utiliza el sistema de gestión editorial OJS (Open Journal Systems), de ahí que todos los manuscritos enviados por los autores deben ser ingresados en este sistema. Para ello, los autores necesitan registrarse y enviar sus artículos acompañados del formulario de Declaración de autoría, buenas prácticas y cesión de derechos. Se devolverá a los autores/as aquellos envíos que no cumplan estas directrices.

2. Los artículos se enviarán a dos árbitros, quienes determinarán en forma anónima: a) publicarlo sin cambios, b) publicarlo cuando se hayan realizado correcciones menores, c) publicarlo una vez que se haya efectuado una revisión a fondo o d) rechazarlo. En caso de discrepancia entre ambos resultados, el texto se enviará a un tercer árbitro, cuya decisión definirá su publicación. Los resultados del proceso de dictamen académico serán inapelables en todos los casos. Los trabajos enviados por académicos serán siempre sometidos a consideración de árbitros externos a su institución.

3. Una vez aceptado el trabajo en su versión final, el autor cede todos los derechos patrimoniales sobre su obra por tiempo indefinido y otorga consentimiento para que The Anáhuac Journal lo reproduzca en distintos medios.

4. Los trabajos autorizados por los árbitros se publicarán en la revista y se notificará a sus autores sobre esta decisión en un plazo máximo de cuatro meses a partir de la recepción del documento.

5. La revista se reserva el derecho de hacer los cambios editoriales que considere pertinentes.

\section{Requisitos y aspectos formales para la presentación de los trabajos}

Como parte del proceso de envío, los autores/as están obligados a comprobar que su envío satisfaga todos los elementos que se muestran a continuación. Se devolverá a los autores/as aquellos envíos que no cumplan estas directrices. Por políticas editoriales, todos los envíos deben hacerse en esta plataforma, por lo tanto, no se recibirán manuscritos enviados directamente a través de correo electrónico. 


\section{Estructura mínima del trabajo:}

- Formato WORD (formato.doc o .docx), tipografía Arial de 12 puntos, interlineado de $1.5 \mathrm{~cm}$.

- La extensión máxima es de 25 hojas tamaño carta (215.9 x $279.4 \mathrm{~mm})$. incluyendo tablas, figuras, referencias y apéndices.

- Los artículos pueden ser escritos en español o inglés y deben incluir un resumen en ambos idiomas. En todos los casos, incluyendo los artículos en español, la calidad del estilo de la versión final es total responsabilidad del autor o autores.

- El resumen será de hasta 180 palabras; deberá incluir máximo 5 palabras clave y la clasificación JEL (Journal of Economic Literature), que puede descargarse de la siguiente liga: https://www.aeaweb.org/econlit/jelCodes.php?view=jel

- El título, resumen y palabras clave deben ir al comienzo del artículo.

- Deberá incluir una introducción que refleje con claridad los antecedentes del trabajo, su desarrollo y conclusiones.

- Las notas de pie de página deberán estar en la hoja correspondiente y deberán usarse para aclarar conceptos o cuestiones editoriales, pero no para las referencias bibliográficas.

- Las referencias bibliográficas se harán según las normas de la APA. No deben extenderse de modo innecesario y deberán aparecer completas en páginas separadas, ordenadas alfabéticamente y, para cada autor, en orden cronológico, del más antiguo al más reciente. Deben tener la información completa sobre la fuente respectiva, incluyendo el DOI (Digital Object Indentifier) cuando esté disponible, y deben insertarse al final del artículo, antes de cualquier apéndice. Los autores deben asegurarse de que haya una correspondencia estricta entre los nombres y años reconocidos en el texto y aquellos listados en la bibliografía, es decir, todos los trabajos citados deberán aparecer en las referencias bibliográficas.

- Las referencias bibliográficas se harán según las normas de la APA que establecen, entre otras, lo siguiente:

a) Libros: Autor (apellido e inicial del nombre). Año de publicación (entre paréntesis). Título del libro (en cursivas), Edición (entre paréntesis y solo si se considera importante). Lugar de edición (:), Editorial. Si no tiene editorial se escribe [s.n.], del latín sine nomine, que significa «sin nombre».

Ejemplo: Castel, R. (1997). Las metamorfosis de la cuestión social. Una crónica del asalariado ( $1^{\mathrm{a}}$ ed.). Argentina: Paidós.

Libro con más de un autor: Autor(es) (apellido e inicial del nombre). Año de publicación (entre paréntesis). Título del libro (en cursivas). Edición (entre paréntesis), Lugar de edición (:), Editorial: De Mattos, C. y Ducci, M.E. (2005). Santiago en la globalización: ¿una nueva ciudad? ( $2^{\mathrm{a}}$ ed.). Santiago: Lom.

Ejemplo: De Mattos, C. y Ducci, M.E. (2005). Santiago en la globalización: ¿una nueva ciudad? (2da ed.). Santiago: Lom.

Note que si el libro está escrito por más de 5, deberá indicarse con un et al. después del primer autor (del latín et alia (y otros).

Ejemplo: Dellanegra, G. et al. (1983). Los países del Atlántico Sur: geopolítica de la Cuenca de la Plata. Buenos Aires: Pleamar.

b) Artículo de revista impresa: Apellido, inicial del nombre. Año de publicación (entre paréntesis). Título del artículo en redondas y entre comillas. Nombre de la 
revista (en cursivas), Volumen de la revista, número de edición (entre paréntesis): intervalo de páginas en el que se encuentra el artículo.

Ejemplo: Oszlak, O. (2009). "El Estado transversal". Encrucijadas UBA, 8 (26): 2-4.

c) Artículo de revista en internet: Apellido, inicial del nombre. Año de publicación (entre paréntesis). Título del artículo (en redondas y entre comillas). Nombre de la revista (en cursivas). Volumen de la revista, número de edición (entre paréntesis), Recuperado de (seguido de dos puntos): página de internet. Ejemplo: Gadner, H. (1983). "La teoría de las inteligencias múltiples". Revista Española de Investigación en Educación, 9 (2). Recuperado de: http://urlinventada.es

Cualquier otra fuente, como base de datos o enciclopedia en línea debe integrar datos detallados del texto citado: autor si lo hay, si no, directamente el título del texto, año si lo hay y si no lo hay, colocar (s/f) (sin fecha). Y la dirección de internet. Recuperado de: página de internet

En el caso de que el texto tenga DOI, incluirlo. Esta es la página para búsqueda de DOI: http://search.crossref.org/?q=

Ejemplo: Morey, C. C. et al. (2015). "The color-sharing bonus: Roles of perceptual organization and attentive processes in visual working memory". Archives of Scientific Psychology, 3: 18-29. DOI: doi.org/10.1037/arc0000014

- Las ilustraciones, fotografías, mapas, diagramas, dibujos, deben tener sus leyendas correspondientes, títulos, una numeración consecutiva, la fuente de donde se obtuvo la información (en caso de ser elaboración propia, hay que señalarlo de esa manera) y deben estar libres de derechos. En caso de imágenes, ilustraciones o dibujos deben estar en alta resolución (300 dpi); en caso de tablas y gráficas deberá enviarse aparte el archivo original en el que fueron creadas (excel, power point, etcétera).

- Los títulos de cuadros y gráficas deben realizarse en tablas de word o excel e ir secuenciados en números arábigos. El cuadro o gráfica debe llevar encabezado en letra negrita tipo Times New Roman y al centro. En la parte inferior debe anotarse la fuente con letra de 10 puntos. En los cuadros debe citarse la fuente (autor y año); con el siguiente formato: bordes (líneas) internos y sencillos, sin bordes izquierdo y derecho en los títulos de cada columna. Las gráficas con borde suave y las barras y líneas en tonos grises. OJO: las fuentes de tablas o gráficos que no sean elaboración propia deben también ir incluidos en las Referencias bibliográficas.

- Las pruebas matemáticas largas y tablas muy detalladas y extensas deberán estar en un apéndice o, en su caso, omitirse. Los autores deberán hacer un esfuerzo por explicar los resultados del significado de las pruebas matemáticas.

- Las ecuaciones deberán presentarse en líneas separadas y centradas. Deberán estar numeradas consecutivamente, en el margen derecho, usando números arábigos entre paréntesis.

- Información del autor(es): en la última página deberán incluirse los datos generales del autor (es): nombre completo, centro o departamento al que se encuentra(n) adscrito(s) laboralmente, dirección postal institucional, dirección de correo electrónico y un breve resumen de su experiencia académica (no mayor a 250 palabras).

- Los manuscritos deben ir acompañados de la carta firmada de aviso de privacidad y cesión de derechos a la revista The Anáhuac Journal. 


\section{Aviso de derechos de autor/a}

(C) 2019, Facultad de Economía y Negocios, Universidad Anáhuac. Reservados todos los derechos. La publicación del artículo en versión impresa implica la cesión total de los derechos de autor (copyright) a la Facultad de Economía y Negocios de la Universidad Anáhuac México. La revista se reserva el derecho para la reproducción total o parcial del trabajo en otros medios impresos, electrónicos o cualquier otra alternativa, reconociendo siempre su autoría.

\section{Declaración de privacidad}

Los datos personales introducidos en esta revista se usarán exclusivamente para los fines establecidos en ella y no se proporcionarán a terceros o para su uso con otros fines.

\section{Acerca de este sistema de publicación}

Esta revista utiliza Open Journal Systems (https://openjournalsystems.com), que es un gestor de revistas de acceso abierto y un software desarrollado, financiado y distribuido de forma gratuita por el proyecto Public Knowledge Project sujeto a la Licencia General Pública de GNU. 